\title{
Anomalous Hopping Exponents of Ultrathin Films of Metals
}

\author{
N. Marković, ${ }^{*}$ C. Christiansen, D. E. Grupp, ${ }^{\S}$ A. M. Mack, ${ }^{\circledR}$ G. Martinez-Arizala, and A. M. Goldman \\ School of Physics and Astronomy, University of Minnesota, Minneapolis, \\ $M N$ 55455, USA
}

(December 18, 1999)

The temperature dependence of the resistance $R(T)$ of ultrathin quench-condensed films of $\mathrm{Ag}, \mathrm{Bi}, \mathrm{Pb}$ and $\mathrm{Pd}$ has been investigated. In the most resistive films films, $R(T)=$ $R_{0} \exp \left(T_{0} / T\right)^{x}$, where $x=0.75 \pm 0.05$. Surprisingly, the exponent $x$ was found to be constant for a wide range of $R_{0}$ and $T_{0}$ in all four materials, possibly implying a consistent underlying conduction mechanism. The results are discussed in terms of several different models of hopping conduction.

\section{INTRODUCTION}

In highly disordered materials, electrical conduction occurs by the hopping of electrons between localized sites. This results in a thermally activated electrical resistance of the form:

$$
R(T)=R_{0} \exp \left(\frac{T_{0}}{T}\right)^{x}
$$

where $T$ is temperature, and $R_{0}, T_{0}$ and $x$ are constants which depend on the disorder, the details of the interactions and the dimensionality of the system. Simple activated hopping over a constant barrier results in the Arrhenius form with $x=1$. For noninteracting electrons, when the average hopping distance depends on temperature due to the compromise between hopping to sites which are close in energy, but farther away, Mott variable range hopping [1] is expected, with $x=1 /(d+1)$, where $d$ is the dimension. Efros and Shklovskii (ES) showed that including Coulomb interactions between electrons results in a soft gap in the density of states at the Fermi energy, which changes the variable range hopping exponent to $x=1 / 2$ in all dimensions [2].

Hopping conduction has been investigated in a wide variety of materials, such as doped semiconductors [3],4], semiconducting heterostructures [5], amorphous metals [6] 10], magnetic materials [11] and superconductors [12]. Both the Mott and the ES forms of variable range hopping have been observed, as well as a crossover between the two regimes [5, 6]. It should be emphasized, however, that it is often hard to distinguish between Mott and ES hopping, particularly in experiments in which the resistance changes only by one or two orders of magnitude. The unambiguous identification of the Mott or ES hopping can be further complicated by factors which are usually neglected, such as the granularity of the system, possible temperature dependence of $R_{0}$, or correlations between electron hops.

While investigating the transport properties of ultrathin quench-condensed films over the course of many years, we have often found that the resistance of the thinnest films was thermally activated with $x \simeq 0.75$. A similar hopping exponent has been reported by other authors [5, 10,13,14, but has rarely been discussed per se. Since there is no theory which predicts this value of the hopping exponent, its origin has been left an open question. Here we report a detailed study of the temperature dependence of the resistance in very disordered films of four different materials: $\mathrm{Ag}, \mathrm{Bi}, \mathrm{Pb}$ and $\mathrm{Pd}$. The films were grown in separate runs over several years, in different cryostats and on different substrates, yet they all show the same thermally activated resistance with an almost identical exponent. A careful analysis of the data points to a new conduction mechanism in this regime, or perhaps calls for a modification of the conventional picture. We compare our results with those of other experiments and available theoretical calculations, and suggest that the model that may possibly explain the anomalous hopping exponent is the collective variable range hopping model of Fisher et al. [15]. Developed to describe vortices in superconductors, this approach has not been considered before in the context of charge transport in disordered electronic systems.

In Section II we survey the various models that have been considered in the discussion of transport in disordered films. We also exhibit the mapping of the model for collective vortex hopping onto the problem of charge transport in disordered systems and estimate the value of the hopping exponent. Experimental details of film growth and resistance measurements are given in Sec. III. In Sec. IV, we analyze the temperature dependence of the film resistance using several different methods to show that the exponent obtained is really a property of the system, rather than a consequence of an improper fit. The results are discussed and compared with other experimental and theoretical work in Sec. V.

\section{SURVEY OF HOPPING MODELS}

In recent years, extensions of the basic variable range hopping model to include percolation effects and correlations between electron hops have been developed. Deutscher et al. 16 proposed a hopping mechanism which leads to a thermally activated resistance with $x$ 
close to $1 / 2$ without considering Coulomb interactions. The mechanism was based on the superlocalization property of wavefunctions on incipient percolation clusters [17], and may be relevant for atomically disordered systems as well as for granular percolative structures. The detailed microstructure of ultrathin quench-condensed films is still not known, and although these films are usually considered to be homogeneous, they may actually contain small grains or clusters. It is then possible that the electrons are restricted to move on a sublattice which is fractal over some range of length scales, and that their wavefunctions decay faster then exponentially with distance. Based on this assumption, a hopping conductivity law has been derived 16 near the percolation threshold, which has the form of Eq 1 with $x=3 / 7$. This is experimentally almost indistinguishable from the Efros-Shklovskii law with $x=1 / 2$ if only the temperature dependence of the resistance is studied, but can be identified through the behavior of the parameter $T_{0}$ and the nature of the crossover to the conventional Mott regime [16].

Generally speaking, there is no reason to assume that the prefactor $R_{0}$ in Eq. 1 is independent of temperature. Van Keuls et al. [5] studied the resistivity in a gated $G a A s / A l_{x} G a_{1-x} A s$ heterostructures as a function of temperature, electron density and magnetic field. Assuming the prefactor of the form:

$$
R_{0}=b T^{m}
$$

where $b$ and $m$ are constants, these workers fit Eq. 1 to their data with $x=1 / 3$ in low magnetic fields and with $x=1 / 2$ in high magnetic fields. The same crossover was observed as a function of electron density and temperature, and it scaled with the separation between the electron layer and the nearby screening gate, as predicted by Aleiner and Shklovskii [18]. In addition to introducing a temperature dependent prefactor, this experiment also raised the issue of the importance of correlations between the electron hops.

In an electron glass, where the screening length is long 19] and the interactions long-ranged, electron hopping may be correlated [20]. Excitations can leave the system far from equilibrium 21] and relaxation occurs through the rearrangement of charge. The energy of a single electron hop may then be significantly reduced by the motion of the surrounding charges. At sufficiently low temperatures, such collective hopping might be the dominant conduction mechanism.

In the analysis of their data, Van Keuls et al. [5] assumed that the number of configurations of occupied states reached by the correlated hopping of a number of electrons is proportional to the single-particle density of states. In that case, the qualitative behavior of the resistance remains unchanged, and the effects of correlations enter through the constants which determine the parameter $T_{0}$ in different regimes.

Yet another issue which can be relevant in extremely thin films is the possibility that the electrons might interact logarithmically rather than as $1 / \mathrm{r}$. As shown by Keldysh [22], the range of the logarithmic interaction is given by:

$$
r_{\log }=\frac{\kappa}{\kappa_{s}+1} d
$$

where $\kappa$ and $\kappa_{s}$ are the dielectric constants of the film and the substrate, respectively, and $d$ is the film thickness. One possible consequence of the logarithmic electronelectron interactions is that the Coulomb gap in the density of states (linear in 2D) may change to an exponential form [23]. This leads to a modified variable range hopping law with a temperature dependent exponent.

Alternatively, the behavior of the logarithmically interacting electrons might be similar to that of vortices in $2 \mathrm{D}$ superconductors, which are known to interact logarithmically. Collective variable range hopping of vortices in disordered thin-film superconductors was studied by Fisher et al. [15]. Including the effect of correlations, these authors found that multivortex hopping results in a lower energy than single vortex hopping. They also suggested that such multiparticle hopping might dominate single particle hopping even in the case of inverse power law interactions.

This approach may then be mapped onto a disordered two-dimensional system of charges. Following the arguments of Fisher et al. [15], the energy $U(r)$ of a multiparticle excitation of length $r$, can be estimated to be:

$$
U(r) \approx K\left(\frac{l}{r}\right)^{1 / 2}
$$

where $l$ is the distance between charges, and $K$ is the bare single-particle excitation energy. The latter is equal to $e^{2} / \kappa a$, where $a$ is the localization length and $\kappa$ is the dielectric constant. The simultaneous hopping of many charges may then result in a lower energy than the hop of a single charge.

The electrical resistance is a product of the probability for an electron to tunnel a distance $r, \Gamma_{r}$, and the probability for an excitation with the energy $U(r)$ to occur, $\Gamma_{U}$ :

$$
R \propto 1 / \Gamma_{r} \Gamma_{U}
$$

A lower bound for the multihop rate can be estimated as follows: if all of the $(r / l)^{2}$ electrons in the $r$ by $r$ region hop a distance comparable to the spacing $l$, then the rate should be proportional to the single-hop rate, $\exp (-l / a)$, raised to the power of the number of electrons, resulting in:

$$
\Gamma_{r} \propto e^{-(l / a)(r / l)^{2}}
$$

The probability for an excitation of energy $U(r)$ is proportional to $\exp (-U(r) / T)$, or using Eq. 迆:

$$
\Gamma_{U} \propto e^{-(K / T)(l / r)^{1 / 2}}
$$


The minimum resistance is obtained when the hopping distance $r_{h o p}$ is:

$$
r_{\text {hop }}^{2} \approx a l\left(\frac{T_{0}}{T}\right)^{4 / 5}
$$

where

$$
T_{0}=K(l / a)^{1 / 4}
$$

Substituting Eqs. 6 through 9 back into Eq. 11 results in a hopping form such as that of Eq. 1 with $x=4 / 5$.

In the other limit, the minimum number of electrons participating in a collective hop could be taken as $(r / d)$, which leads to $x=2 / 3$. The collective variable range hopping mechanism may therefore result in a resistance of the form of Eq. 1, where the range of exponents is $2 / 3<x<4 / 5$, depending on the fraction of electrons participating in the process.

\section{EXPERIMENTAL METHODS}

The temperature dependence of the resistance has been studied in ultrathin quench-condensed films of $\mathrm{Ag}, \mathrm{Bi}, \mathrm{Pd}$ [8] and $\mathrm{Pb} 24$ 25]. The films were deposited on liquid helium cooled substrates and resistance measurements were performed in situ at temperatures down to $0.15 \mathrm{~K}$. Ultra-high vacuum conditions and temperatures below $20 K$ were sustained throughout each run, in order to avoid contamination or crystallization. The substrates were glazed alumina (for $\mathrm{Bi}$ and $\mathrm{Pd}$ films) or $\mathrm{SrTiO}_{3}$ (100) (for $\mathrm{Ag}$ and $\mathrm{Pb}$ films). The $\mathrm{SrTiO}_{3}$ (100) substrates were $0.75 \mathrm{~mm}$ thick and had a $100 \mathrm{~nm}$ thick $\mathrm{Au}$ gate on the back. Such a gate can be used to study the response of the film to a perpendicular electric field and was used to establish glass-like behavior in the most resistive films [24]25].

Films were deposited in thickness increments between 0.05 and $0.5 \AA$ on top of a thin germanium layer $(5-10 \AA)$. (The Pd films were the exception to this as they were deposited directly onto glazed alumina substrates where they became connected at monolayer coverage.) Films grown on amorphous Ge are believed to be homogeneous, since they become connected at an average thickness of about one monolayer [26]. The thicknesses of the films studied ranged from $5 \AA$ up to $15 \AA$. These nominal values of the film thickness are calculated from the deposition rate, which was measured using a quartz crystal monitor placed in the vicinity of the substrate. The first low-temperature scanning tunneling microscopy studies of the morphology of films grown in a similar manner indicate that the thinnest films may indeed be homogenous, while the thicker ones contain small clusters [27].

Resistance measurements were carried out using a standard dc four-probe technique. Very low bias currents $(<10 n A)$ were used to avoid Joule heating of the sample and to make sure that the voltage across the sample was a linear function of the applied current. When measuring very resistive films $\left(10^{4}-10^{8} \Omega\right)$, because of the long time constants of the circuit, it was necessary to monitor the voltage as a function of time after the current was changed, and allow adequate time for the voltage to stabilize. To avoid the voltage offset errors due to thermal EMFs, both polarities of the current were used to determine the resistance.

The resistance of a series of $\mathrm{Ag}$ films was also studied in a magnetic field. Magnetic fields of up to 20kG (12 $\mathrm{kG}$ ) were applied in direction parallel (perpendicular) to the plane of the substrate using a split-coil superconducting magnet. In a regime where the anomalous hopping exponent is observed, the resistance was found to be independent of magnetic field.

\section{RESULTS AND ANALYSIS}

The temperature dependence of the sheet resistance (resistance per square) for series of $\mathrm{Ag}, \mathrm{Bi}, \mathrm{Pb}$ and $\mathrm{Pd}$ films is shown in Fig. 11. The logarithm of the sheet resistance, plotted as a function of $T^{-0.75}$ follows a straight line for each film, indicating that the resistance is thermally activated with $x \approx 0.75$. Using other values for $x$, such as $1,1 / 2$ or $1 / 3$ yielded considerably larger deviations from the data.

Since the prefactor in Eq. I is generally expected to be temperature-dependent $(m \neq 0$ in Eq. 2), we attempted to fit the data using different combinations of $m$ and $x$. As shown in Fig. 2, using values of $m$ greater than zero actually increased the error of the fit. The maximum deviation in the fit of the combinations of Eqs. 1 and 2 to the data became much larger than the noise in $R$ as $m$ was increased. Furthermore if values of $m$ were chosen to force either Mott or ES hopping exponents of $x=1 / 3$ or $1 / 2$, respectively, the quality of fits as measured by chi squared would be significantly worse than that with $m=0$, in contrast with the findings of Van Keuls et al. [5.

Assuming that the hopping exponent is $x \approx 0.75$, the activation energy $T_{0}$ can be extracted from the fit to Eq. 1. The values of the parameter $T_{0}$ for different films of all four materials are shown in Fig. 3 as a function of $R_{14 K}$, which is the sheet resistance measured at $14 K$. This quantity is inversely proportional to the film thickness, so by using $R_{14 K}$ instead of the thickness, one can avoid systematic errors in the nominal thicknesses of the films of different materials. It is apparent in Fig. 3 that $T_{0}$ changes greatly as $R_{14 K}$ (and therefore also the film thickness) changes, ranging from around $100 \mathrm{~K}$ for the thinnest films, to around $10 \mathrm{~K}$ for the thickest films. The same qualitative and quantitative behavior was found for all four materials.

A more direct method of determining the exponent $x$ (which is exact under the condition $m<<\left(T_{0} / T\right)^{x}$ ) has been developed by Zabrodskii and Zinov'eva [ 1 . 
The method is based on defining the function $w=$ $-d(\log R) / d(\log T)$. If $R$ is given by Eq. 1, then $\log w \propto$ $-x \log T$. By plotting $\log w$ as a function of $\log T, x$ can be easily extracted from the slope of the resulting straight line. The benefit of the Zabrodskii-Zinov'eva approach is the simplicity of fitting a line rather than a complicated function with up to four adjustable parameters $(b$, $m, T_{0}$ and $x$ ). Once it has been determined that $m=0$, this method eliminates the danger of finding a local minimum instead of the best fit. The results of determining $x$ this way are shown in Fig. A. For very resistive films, plotting $\log w$ vs. $\log T$ indeed yielded straight lines. The values of $x$ varied slightly between different materials, from $x \approx 0.7$ for Ag to $x \approx 0.8$ for Bi. Remarkably, the value of $x$ did not change between different films in the same series over a significant range of sheet resistances, as shown in Fig. 5 .

Even though $R_{14 K}$ and $T_{0}$ change from film to film, as more material is added to increase the average thickness and decrease the sheet resistance of the film, $x$ stays constant over three orders of magnitude in $R_{14 K}$. For thicker films (smaller $R_{14 K}$ ), $x$ drops rather abruptly to a value between $1 / 3$ and $1 / 2$. In this regime, the ZabrodskiiZinov'eva plots no longer produce straight lines, indicating that the hopping exponent changes as a function of temperature. Further increase of the film thickness leads to another crossover to a weakly localized regime where the temperature dependence of the resistance is logarithmic (not shown in Fig. 5).

\section{DISCUSSION}

An activated temperature dependence of the resistance with an anomalous hopping exponent $x \approx 0.75$ has been observed in disordered films of four different materials, grown on different substrates and measured in different cryostats. This strongly suggests that the exponent $x \approx$ 0.75 is a general property of ultrathin films of metals in the very strongly localized regime.

The same exponent has been reported by Adkins and Astrakharchik 110 in ultrathin quench-condensed films of $\mathrm{Bi}$ with a $\mathrm{Ge}$ underlayer. In that experiment, the temperature dependence of the resistance changed to simply activated (with $x=1$ ) when the Coulomb interaction was screened in the presence of a nearby metallic gate. This behavior was ascribed to the fixed range hopping of dipoles in screened films, but no details were given on the origin of $x \approx 0.75$ in unscreened films. The authors suggest that the films may be in the crossover regime between the variable range hopping and the fixed range hopping regime. Such a crossover can occur when the optimal hopping distance $r_{h o p}$ becomes comparable with the localization length $\xi$.

In our experiment, $x$ stays constant over several orders of magnitude in sheet resistance, and then drops abruptly as the sheet resistance decreases further. If our films were merely at the crossover between $x \approx 1 / 2$ (or $1 / 3$ ) and $x \approx 1$, the change in $x$ would be expected to be gradual. The observed constancy of $x$ implies that a consistent mechanism may be governing the conduction in this regime, a mechanism different from Mott or Efros-Shklovskii variable range hopping which is usually observed in less resistive films.

Furthermore, it was not possible to obtain a satisfactory fit to the data using a temperature-dependent prefactor, as in the work of Van Keuls et al. It is interesting that these workers obtain $x \approx 0.75$ in all magnetic fields if $m$ is taken to be zero. However, the activation energies obtained from such fits are reported to be unacceptably small [5].

There are several other mechanisms which may be relevant in a very disordered $2 \mathrm{D}$ system. For example, Dai et al. [3] observed an exponent $x \sim 1$ in $\mathrm{Si}: \mathrm{B}$, which changed to $x \sim 1 / 2$ when a magnetic field was applied. They suggested that the $x \sim 1$ was due to the exchange interaction between the electron spins, which is destroyed in a magnetic field. It must be noted that the $\mathrm{Ag}$ did not show any magnetoresistance up to the highest field available, $20 \mathrm{kG}$, so the exchange interaction is most likely not the origin of the anomalous hopping exponent observed in these films.

If we allow a possibility that our films are granular on a very small scale (which we cannot unequivocally rule out), than we must consider the superlocalization mechanism of Deutscher et al. [16] as a possible candidate to explain our data. Without Coulomb interactions, this model predicts $x \approx 0.43$, which obviously cannot account for our results. Including the Coulomb interactions may lead to a higher exponent, as proposed by van der Putten et al [14. These authors studied the hopping conductivity of percolating carbon-black-polymer compounds and found $x \approx 0.66$, which is much closer to our result, although still too low. They interpret their results as evidence of superlocalization on a fractal network with Coulomb dominated hopping. The activation energies were found to be independent of the electron concentration, as predicted by Deutscher et al. In contrast, the activation energies found in our experiment depend strongly on the film thickness, which is closely related to the electron concentration. Furthermore, if the Coulomb interactions were screened, one might expect the exponent to decrease towards 0.43 , rather than to increase towards 1, as observed in screened Bi films by Adkins and Astrakharchik [10].

Another possibility is that the anomalous exponent is a consequence of the exponential gap in the density of states, which can arise if the electrons interact logarithmically 23. In that case, the hopping exponent would be something close to, but smaller than 1 at higher temperatures, and then cross over smoothly to $1 / 2$ at low temperatures. Forcing a fit of Eq. 11 to the data would result in an exponent which changes continuously with temperature. In the less resistive regime where we observe a temperature-dependent exponent, a closer inspec- 
tion shows the opposite trend: the exponent is close to $1 / 2$ at higher temperatures, and increases with decreasing temperature. On the other hand, we cannot rule out the possibility that we might observe a smooth crossover to $1 / 2$ in the most resistive films if we could measure at much lower temperatures.

Finally, we consider the collective variable range hopping mechanism, proposed in the context of vortices in disordered superconductors by Fisher et al. [15]. The mapping of this model onto a $2 \mathrm{D}$ electron system may actually be exact, if the electron-electron interactions are logarithmic over relevant length scales, but the authors suggest that collective hopping may dominate over the single-particle hopping even in the case of a conventional Coulomb interaction. The range of exponents predicted by the collective hopping model is $2 / 3<x<4 / 5$, depending on the ratio of electrons which participate in the process. The exponent found in our experiment, as well as the exponents found by other groups 10,5, 13, 14, are well within that range. The activation energies are expected to depend on the localization length, which in turn depends on the film thickness, as shown in Fig. 3.

In conclusion, we have addressed the issue of the anomalous hopping exponent $x \approx 0.75$ observed in ultrathin films of metals and related $2 \mathrm{D}$ systems. We argue that this hopping exponent is a general property of very strongly disordered systems, rather than a result of an improper fit or a signature of some sort of a crossover behavior. The usual models of hopping conduction do not explain this result. Our data can be explained by a collective variable range hopping mechanism, but our work by no means provides a proof of such a mechanism. Future experimental and theoretical studies will be needed to shed more light on this matter.

We gratefully acknowledge useful discussions with Boris Shklovskii and Leonid Glazman. This work was supported in part by the National Science Foundation under Grant No. NSF/DMR-987681.

* Present Address: Department of Applied Physics, Technical University of Delft, the Netherlands.

$\S$ Present Address: Center for Integrated Systems, Stanford University, Stanford, CA, USA.

ฯ Present Address: Seagate Technology, Bloomington, MN, USA.

[1] N. F. Mott, J. Non-Crystal. Solids 1, 1 (1969).

[2] A. L. Efros and B. I. Shklovskii, J. Phys. C 8, L49 (1975).

[3] P. Dai, Y. Zhang, and M. P. Sarachik, Phys. Rev. Lett. 69, 1804 (1992).
[4] A. G. Zabrodskii and K. N. Zinov'eva, Zh. Eksp. Teor. Fiz. 86, 727 (1984) [JETP 59, 425 (1984)].

[5] F. W. Van Keuls, X. L. Hu, H. W. Jiang, and A. J. Dahm, Phys. Rev. B 56, 1161 (1997).

[6] S. J. Lee, J. B. Ketterson, and N. Trivedi, Phys. Rev. B 46, 12695 (1992).

[7] I. Shlimak, M. Kaveh, M. Yosefin, M. Lea and P. Fozooni Phys. Rev. Lett. 68, 3076 (1992).

[8] Y. Liu, B. Nease, and A. M. Goldman, Europhys. Lett.19, 409 (1992).

[9] S.-Y. Hsu and J. M. Valles, Jr., Phys. Rev. Lett. 74, 2331 (1995).

[10] C. J. Adkins and E. G. Astrakharchik, J. Phys. Condens. Matter 10, 6651 (1998).

[11] A. S. Ioselevich, Phys. Rev. Lett. 67, 2307 (1991).

[12] C. Dekker, P. J. M. Woltgens, R. H. Koch, B. W. Hussey, and A. Gupta, Phys. Rev. Lett. 69, 2717 (1992).

[13] M. E. Gershenson, Yu. B. Khavin, D. Reuter, P. Schafmeister, and A. D. Wieck, cond-mat/9908097.

[14] D. van der Putten, J. T. Moonen, H. B. Brom, J. C. M. Brokken-Zijp, and M. A. J. Michels, Phys. Rev. Lett. 69, 494 (1992).

[15] M. P. A. Fisher, T. A. Tokuyasu, and A. P. Young, Phys. Rev. Lett. 66, 2931 (1991).

[16] G. Deutscher, Y. Lévy, and B. Souillard, Europhys. Lett. 4, 577 (1987).

[17] Y.-E. Lévy and B. Souillard, Europhys. Lett. 4, 233 (1987).

[18] I. L. Aleiner and B. I. Shklovskii, Phys. Rev. B 49, 13721 (1994).

[19] D. Monroe, A. C. Gossard, J. H. English, B. Golding, W. H. Haemmerle, and M. A. Kastner, Phys. Rev. Lett. 59, 1148 (1987).

[20] M. Pollak, Disc. Faraday Soc. 50, 13 (1970).

[21] Z. Ovadyahu and M. Pollak, Phys. Rev. Lett. 79, 459 (1997).

[22] L. V. Keldysh, Pis'ma Zh. Eksp.Teor. Phys. 29, 716 (1979) [JETP Lett. 29, 659 (1979)].

[23] B. I. Shklovskii, private communication, and A. I. Larkin and D. E. Khmel'nitskii, Zh. Eksp.Teor. Fiz. 83, 1140 (1982).

[24] G. Martinez-Arizala, D. E. Grupp, C. Christiansen, A. M. Mack, N. Marković, Y. Seguchi, and A. M. Goldman, Phys. Rev. Lett. 78, 1130 (1997).

[25] G. Martinez-Arizala, C. Christiansen,D. E. Grupp, N. Marković, A. M. Mack, and A. M. Goldman, Phys. Rev. B 57, R670 (1998).

[26] M. Strongin, R. S. Thompson, O. F. Kammerer,and J. E. Crow, Phys. Rev. B 1, 1078 (1970).

[27] K. L. Ekinci and J. M. Valles, Jr., Phys. Rev. B 58, 7347 (1998); Phys. Rev. Lett. 82, 1518 (1999).

FIG. 1. Sheet resistance as a function of $\mathrm{T}^{-0.75}$ for a series of $\mathrm{Ag}, \mathrm{Bi}, \mathrm{Pb}$ and $\mathrm{Pd}$ films. Each curve represents a different thickness, and the film thickness increases from top to bottom:

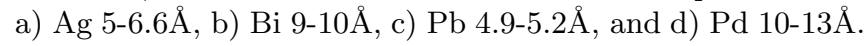


FIG. 2. Best fits for $x$ (full circles) for increasing exponent $\mathrm{m}$ in the resistance prefactor $T^{m}$ in the combination of Eqs. 1 and 2 for a $7.0 \AA$ thick $\mathrm{Ag}$ film. Chi squared (open circles) is a minimum at $m=0$. The deviation of the fit from the data quickly becomes much larger than the noise in $R$ as $m$ is increased.

FIG. 3. The activation energy $T_{0}$ as a function $R_{14 K}$ for different films of all four materials: Ag (crosses), Bi (squares), $\mathrm{Pb}$ (diamonds) and $\mathrm{Pd}$ (circles).

FIG. 4. Zabrodskii plots for a series of $\mathrm{Ag}, \mathrm{Bi}, \mathrm{Pb}$ and $\mathrm{Pd}$ films. Each curve represents a different thickness, and the film thickness increases from top to bottom: a) Ag 5-6.6 b) $\mathrm{Bi} 9-10 \AA$, c) $\mathrm{Pb} 4.9-5.2 \AA$, and d) $\mathrm{Pd} 10-13 \AA$. The slopes determine the exponent $x$.

FIG. 5. The hopping exponent $x$ as a function of the sheet resistance $R_{14 K}$ for all four materials: $\mathrm{Ag}$ (crosses), $\mathrm{Bi}$ (squares), $\mathrm{Pb}$ (diamonds) and $\mathrm{Pd}$ (circles). 

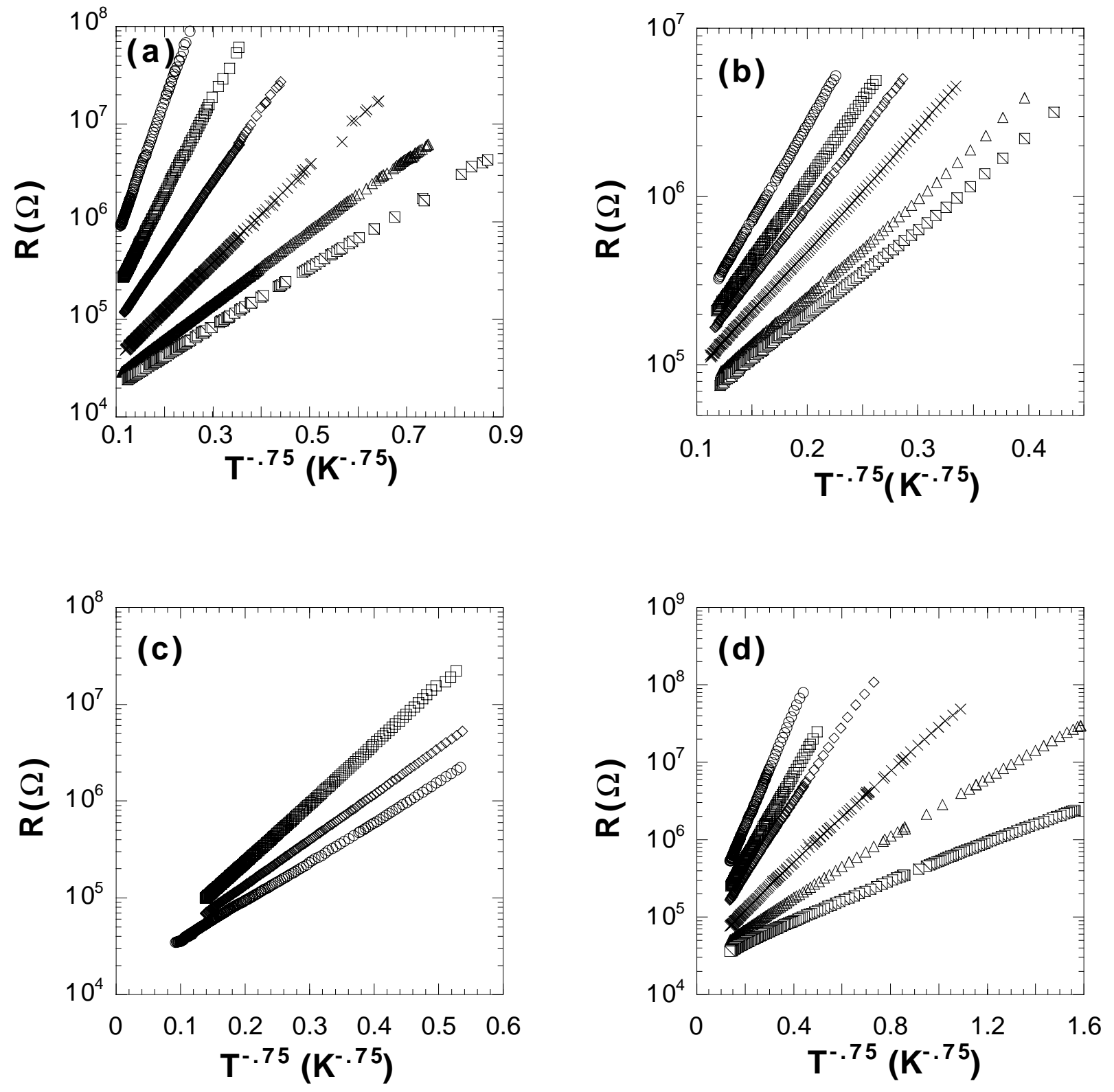


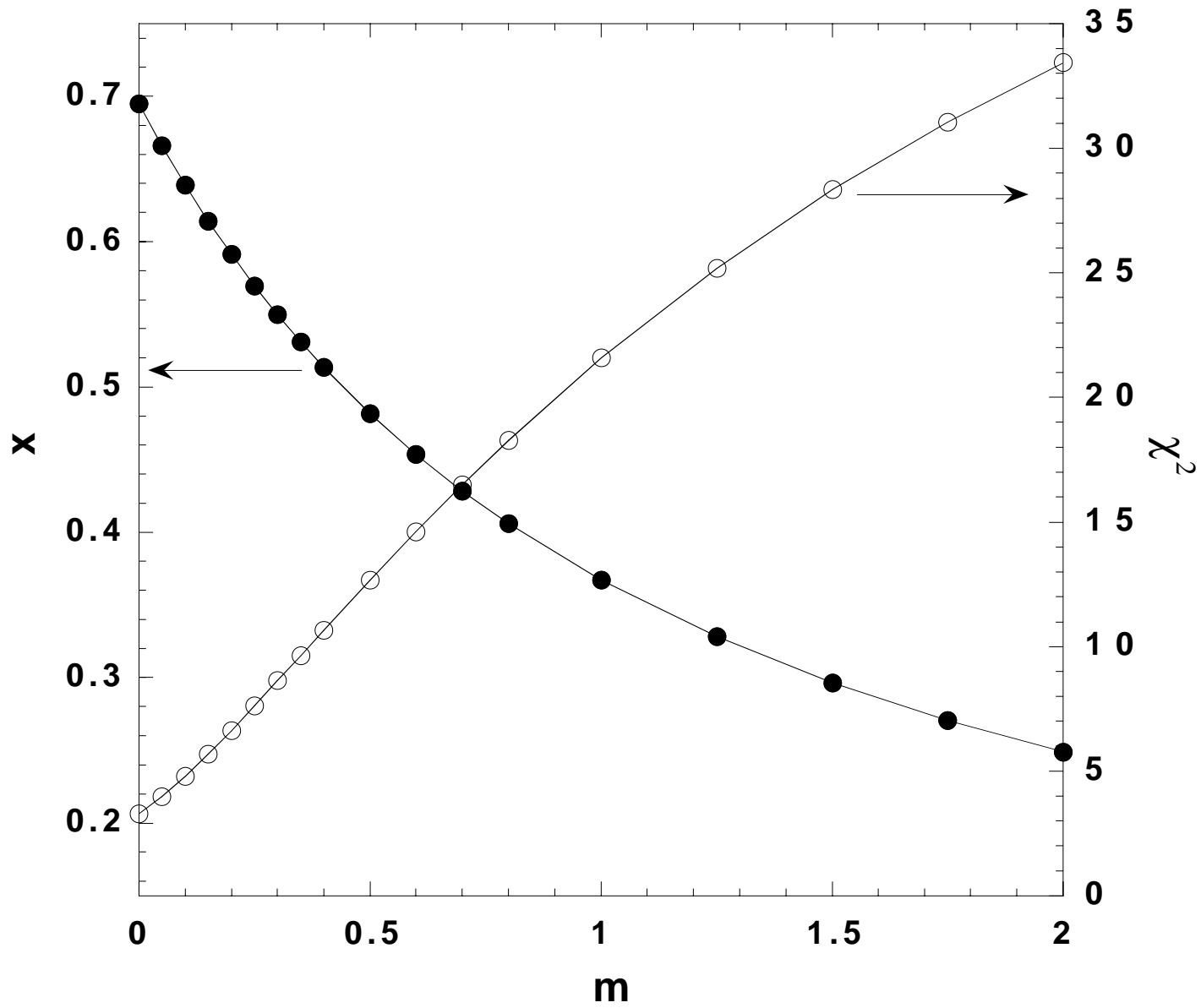




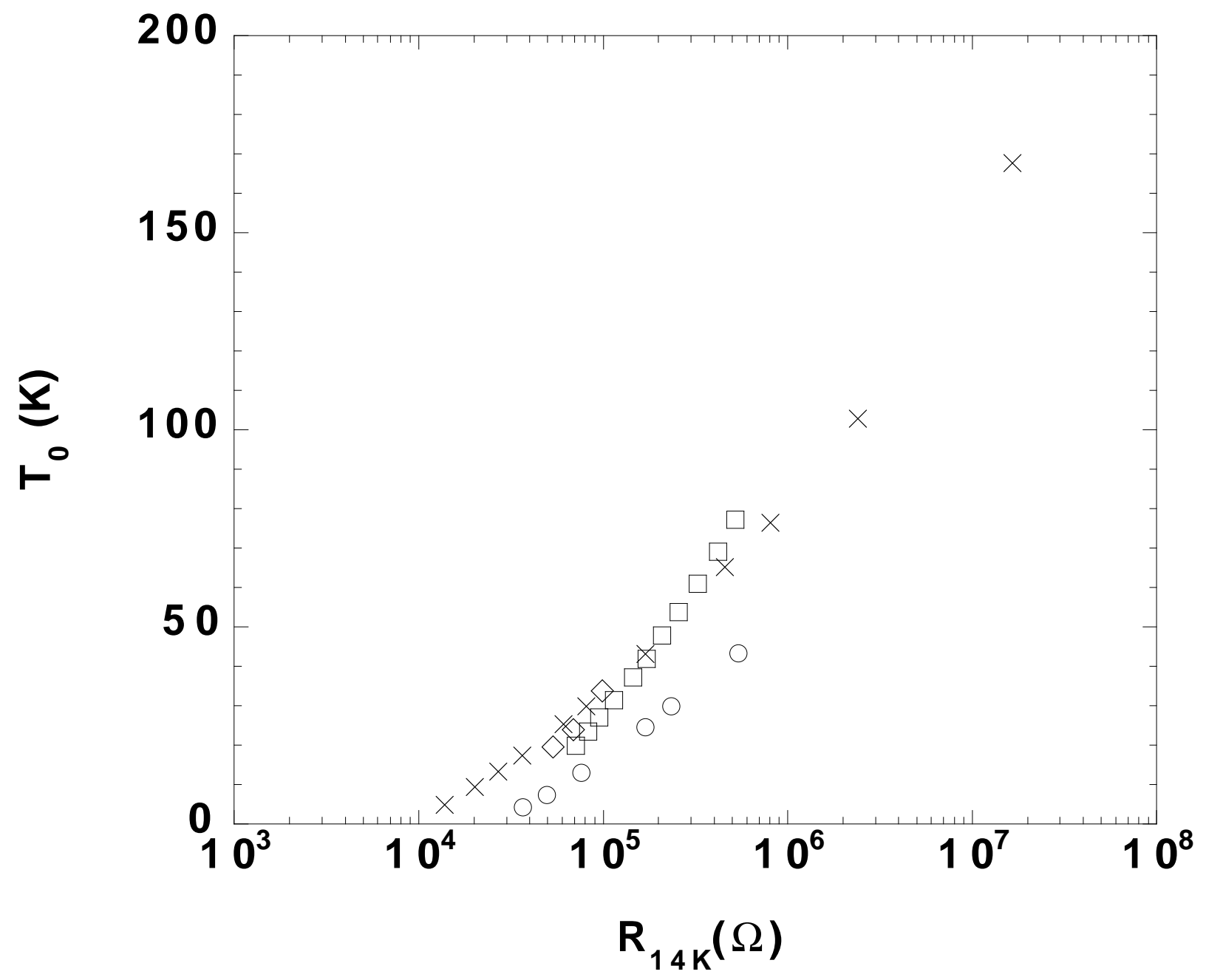



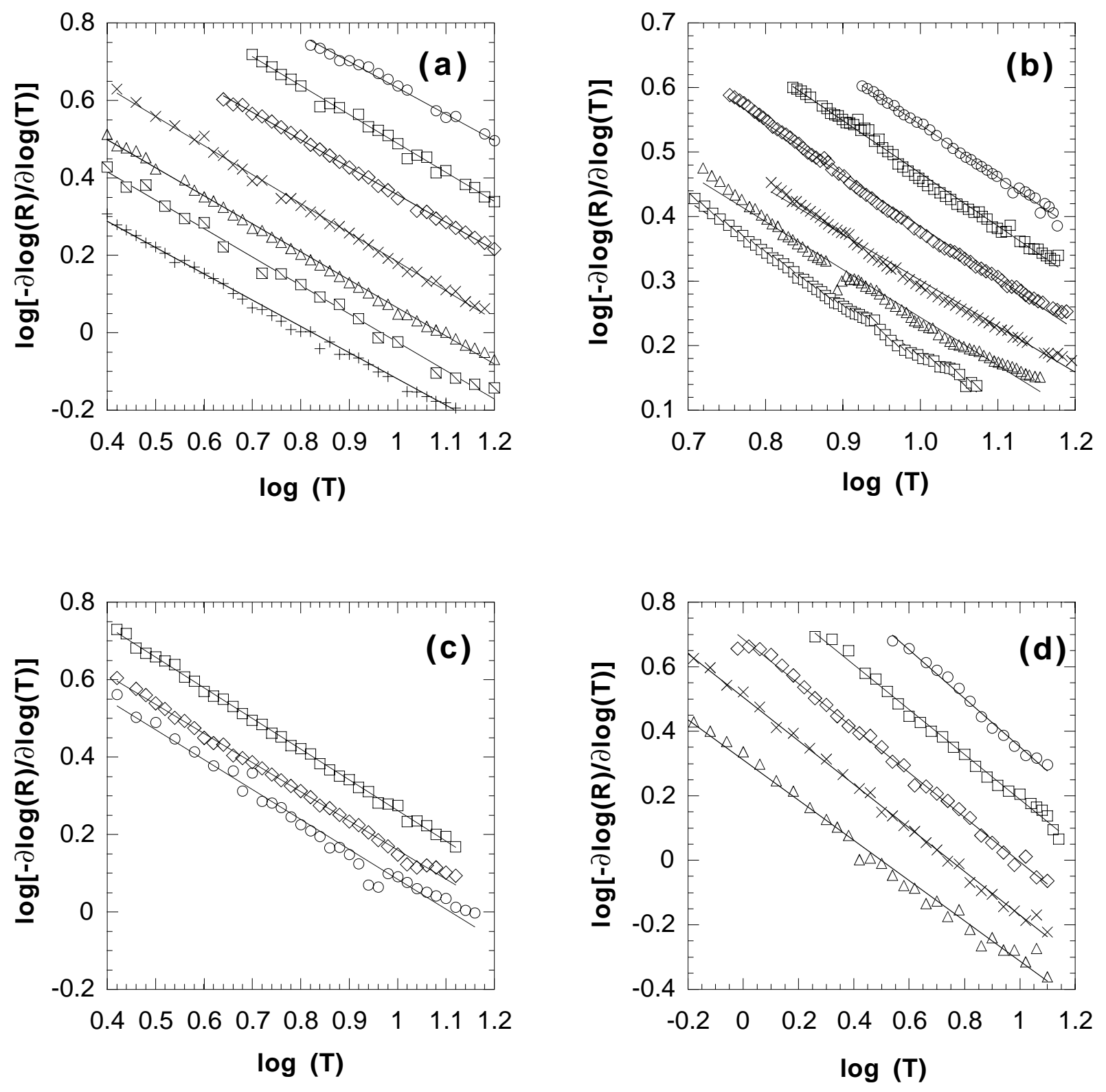


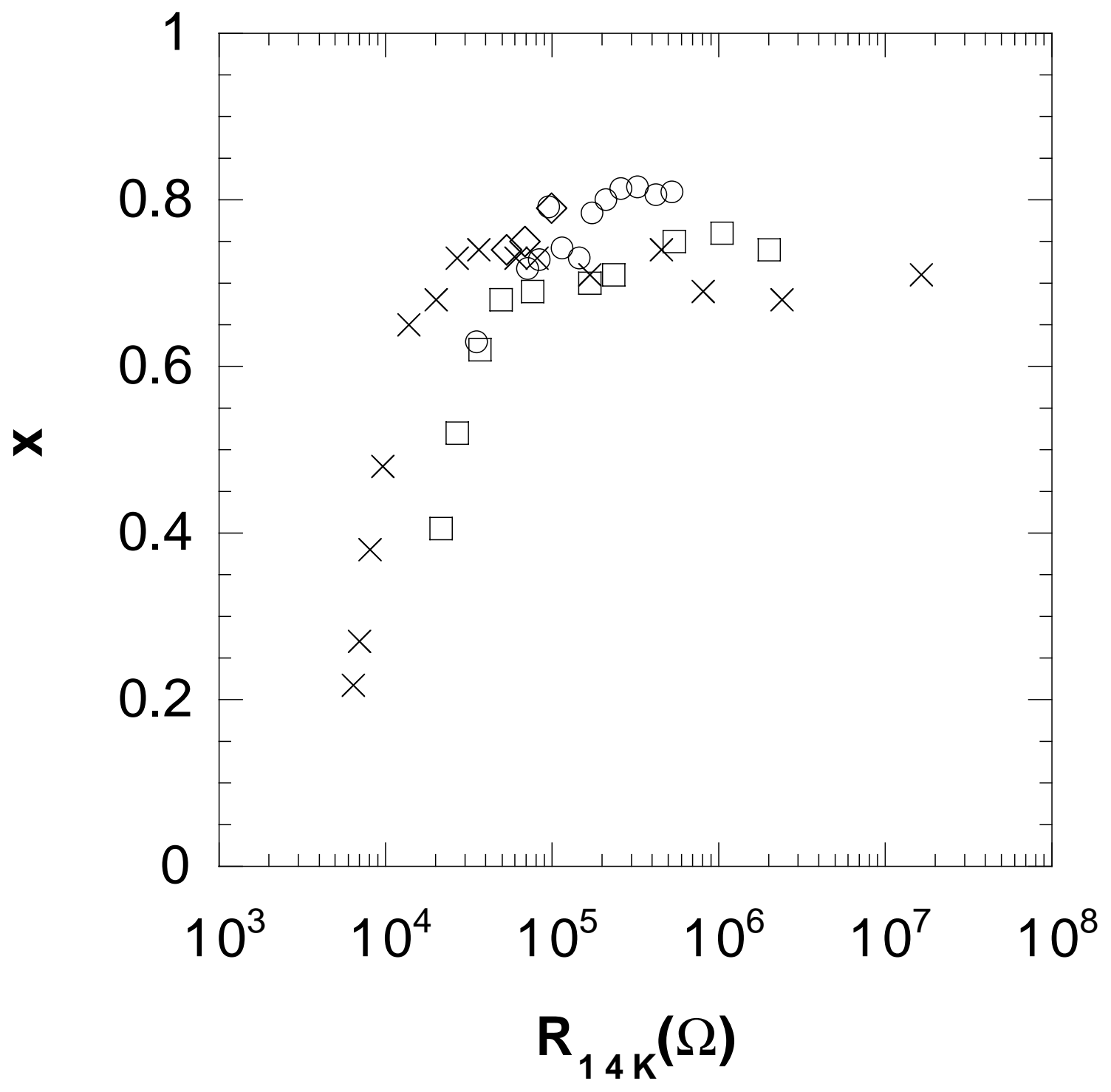

